\section{Raman spectroscopy technology to monitor the carotenoids in skin of thalassemia patients: a novel non-invasive tool relating oxidative stress with iron burden}

\author{
Anna Perrone, ${ }^{1}$ Luisa Tesoriere, \\ Anna Maria Pintaudi, ${ }^{1}$ \\ Alessandro Attanzio, ${ }^{1}$ Paolo Rigano, ${ }^{2}$ \\ Aurelio Maggio, \\ Maria Antonietta Livrea ${ }^{1}$ \\ 'Dipartimento STEBICEF, Università di \\ Palermo; ${ }^{2}$ Divisione di Ematologia e \\ Malattie rare del sangue e degli organi \\ ematopoietici, Ospedali Riuniti Villa Sofia \\ Cervello, Palermo, Italy
}

\section{Abstract}

In this work we approach the relationship between redox state and iron overload by noninvasive instrumental techniques. Intracardiac, liver iron and liver fibrosis have been monitored in transfusion-dependent thalassemia patients by magnetic resonance imaging and hepatic transient elastography examinations. These measurements have been matched with a non-invasive, and yet unexplored in clinical practice, evaluation of body's oxidative stress through measurement of antioxidant carotenoids in skin, by a spectroscopic method based on Raman technology (RRS). The global body's antioxidant status results from a balance between the level of antioxidants in cells and body fluids, including blood, and pro-oxidant species endogenously produced or coming from external sources. On this basis, the level of skin carotenoids can be considered a biomarker of the entire antioxidant status. In our work the use of RRS method provided information on the redox state of thalassemia patients, which was correlated with the iron status of the patients. Due to the highly adverse effects of accumulated iron, the novel, simple, non-invasive RRS to monitor dermal carotenoids with high compliance of the patients may be a useful tool for the management of thalassemia patients.

\section{Introduction}

A characteristic depletion of antioxidant defenses and oxidative stress are associated with hematologic disorders such as $\beta$-thalassemia, ${ }^{1-3}$ with the iron burden to be consid- ered the main reason of the redox imbalance. Frequent blood transfusions and increased intestinal absorption of iron, after saturation of transferrin, indeed favor the accumulation of highly reactive low molecular weight iron in cells (labile iron pool), followed by production of various reactive oxygen species (ROS) through Fenton chemistry, oxidation of cell components, impairment of cell functions, cell death and finally organ damage. Though both liver and heart are principally involved in dramatic iron accumulation, heart failure due to myocardial iron overload is the most dreadful consequence of iron toxicity in thalassemia, because heart has less developed antioxidant defenses with respect to other organs. ${ }^{4}$ In addition to iron overload tissue hypoxia, with damaging mitochondrial activity, will cause formation of ROS, thus contributing to the redox imbalance. $^{5}$

Conventional measurements of blood ferritin and non-transferrin bound iron and of a number of blood parameters relevant to body's redox state may be used to monitor iron burden and oxidative stress of thalassemia patients. Our group first showed that the plasma levels of main antioxidant vitamins (vitamin A, vitamin E, carotenoids) were decreased in thalassemia patients and inversely correlated with both ferritin and indices of liver damage, whereas a remarkably increased level of end-products from peroxidation of lipids and proteins showed a positive correlation with these parameters. ${ }^{1}$ In this work we approach the relationship between redox state and iron overload by non-invasive instrumental techniques. Intracardiac and liver iron, and liver fibrosis, have been monitored in transfusiondependent thalassemia patients by magnetic resonance imaging based $\mathrm{T} 2 *$ multislice measurements, ${ }^{6}$ and by hepatic transient elastography (FibroScan ${ }^{\circledR}$ ) examinations. These measurements have been matched with a non-invasive, and yet unexplored in clinical practice, evaluation of body's oxidative stress through measurement of antioxidant carotenoids in skin, ${ }^{7-10}$ by a spectroscopic method based on Raman technology. ${ }^{11}$

Resonance Raman light scattering spectroscopy (RRS) is a form of laser spectroscopy based on the Raman effect. When a laser monochromatic light interacts with some molecules, these diffuse the light emitting a new, higher wavelength, monochromatic light. Because of their conjugated carbon backbone molecular structure, carotenoids possess characteristic vibrational/rotational energy levels that make them particularly well suited for RRS, strongly absorbing in the blue wavelength region and emitting in the green region. ${ }^{12}$ These properties have first led to explore the use of RRS for the quantitative optical measurement of carotenoids in human tissues, from macula lutea, ${ }^{13-15}$ to oral
Correspondence: Maria A. Livrea, Dipartimento STEBICEF, Università di Palermo, Via M. Cipolla 74. 90123 Palermo, Italy.

E-mail: maria.livrea@unipa.it

Key words: Raman spectroscopy technology, skin carotenoids, thalassemia, oxidative stress.

Received for publication: 4 October 2013.

Revision received: 25 October 2013.

Accepted for publication: 20 May 2014.

This work is licensed under a Creative Commons Attribution 3.0 License (by-nc 3.0).

(C) Copyright A. Perrone et al., 2014

Licensee PAGEPress, Italy

Thalassemia Reports 2014; 4:1967

doi:10.4081/thal.2014.1967

mucosa, ${ }^{16}$ and skin. ${ }^{8,9,17,18}$ The use of RRS for detection and quantitation of skin carotenoids has been validated by matching with extraction and conventional high-performance liquid chromatography (HPLC) measurements of carotenoids in skin specimens and in blood. The assessment of skin carotenoid status by RRS is now considered valid, feasible and reproducible,,$^{10}$ and correlated with the blood carotenoid level. ${ }^{19-21}$ The global body's antioxidant status results from a balance between the level of antioxidants in cells and body fluids, including blood, and pro-oxidant species endogenously produced or coming from external sources. Body's molecular antioxidants work in concert and preserve each other, so the level of each one in the pool reflects the level of the whole antioxidant pool. On this basis, the level of skin carotenoids can be considered a biomarker of the entire antioxidant status. In our work the use of RRS-based portable scanner allowing measurements of carotenoids in the upper $0.1 \mathrm{~mm}$ stratum corneum of skin of the palm of a hand, provided information on the redox state of thalassemia patients, which was correlated with the iron status of the patients.

\section{Materials and Methods}

\section{Subjects and experimental protocol}

Fifty-seven patients (31 with thalassemia major; 26 with transfusion-dependent thalassemia intermedia), 27 females and 30 males, aged 12 to 72 years (mean, $39.4 \pm 14.37$ ), were recruited, with consent, for this study, and were under observation for 1 year. All of the patients had previously been characterized for $\beta$-globin gene mutations. Patients were regularly interviewed and examined by a staff of physicians at 15-30 days intervals. Serum ferritin was measured every month, and peri- 
odic cardiac, hepatologic and endocrinologic evaluations were performed. The patients were transfused (2-4 week intervals) to maintain pre transfusion hemoglobin levels above 9.5-10 g per dL. Twenty patients had undergone splenectomy. Iron chelation therapy was performed with deferasirox in 30 patients, with deferipron in 24 patients and with desferroxamine in 3 patients. Some patients exhibited clinical complications by secondary iron overload: insulin-dependent diabetes mellitus (5 patients), hypothyroidism (1 patient), hypogonadism (17 patients), reduced left-ventricular ejection fraction $<50 \%$ (6 patients). Twenty-four patients were hepatitic $\mathrm{C}$ virus RNA positive, with levels of serum alanine (AST) and aspartate aminotransferases (ALT) 2-3 N. Lycopene was extracted from $500 \mu \mathrm{L}$ serum samples, diluted $1: 2$ with $0.15 \mathrm{mM} \mathrm{NaCl}$, with 1 volume of methanol and 3 volumes of hexane:diethyl ether (l:l, vol:vol). The extracts were then dried under nitrogen, resuspended with a mixture of acetonitrile:methanol: tetrahydrofurane (58.5:35:6.5, vol:vol:vol), and analyzed with the same solvent ${ }^{22}$ by a HPLC Supelco Supelcosil LC-18 column $(0.4625 \mathrm{~cm})$ (Bellefonte, PA, USA), at a flow rate of $2.5 \mathrm{~mL}$ $\min ^{1}$. Under these conditions lycopene eluted at $8.2 \mathrm{~min}$. Revelation was at $450 \mathrm{~nm}$.

Magnetic resonance $\mathrm{T}^{*}{ }^{*}$ technique ${ }^{6}$ was applied to measure cardiac and liver iron concentration in 38 of the patients.

Noninvasive assessment of liver stiffness was performer by transient elastography carried out with FibroScan ${ }^{\circledR}$ in 42 of the patients) and expressed as $\mathrm{kPa}$ correlated to fibrosis.
Measurement of carotenoids in skin

A portable Raman spectroscopy, Pharmanex $^{\circledR}$ Biophotonic Scanner S2 (NuSkin, Provo, UT, USA), designed to monitor carotenoids in the $0.1 \mathrm{~m}$ stratum corneum of the skin of the hand, has been used for the measurements. A low intensity 471.3-473 nm radiation from light emitting diodes interacts with the skin carotenoids. The scattered light is detected at $507.8-509.8 \mathrm{~nm}$ by the scanner that converts the Raman intensity in counts [skin carotenoids score (SCS)]. A computer then transforms the scanner signals in a colored scale going from red (lowest intensity, poor carotenoid score, $<19,000)$ to dark blue (highest intensity, high carotenoid score, $>50,000$ ). All patients underwent skin carotenoid measurement two times with a 4 to 6 months time interval, before transfusion, and SCS data are reported as the mean of two determinations for each patient. Healthy individuals aged 25 to $67(n=60)$ served as control.

\section{Statistical analysis}

All results are expressed as means \pm standard deviation (SD). Comparison between controls and thalassemia patients was performed by the unpaired Student's $t$-test. Pearson's correlations were used to determine relationships between covariates.

\section{Results}

Hematological and instrumental measurements of iron and of parameters of liver damage of thalassemia patients are listed in Table 1. Feasibility of the RRS methodological approach to measure skin carotenoids as a reflection of the plasma concentration in thalassemia patients was first researched. Since lycopene is the major Raman sensitive carotenoid in the skin, ${ }^{9}$ the correlation of the SCS of each patient and his plasma level of lycopene was investigated. Figure 1 shows a net positive correlation between SCS and plasma values, thus validating the use of this spectroscopic method to measure the body carotenoid level even in pathologies characterized by remarkable oxidative injury such as thalassemia. As expected, since licopene reflects the antioxidant imbalance and may be an index of the individual oxidative status, the mean of its plasma level in patients $(0.17 \pm 0.09$ $\mu \mathrm{M}, \mathrm{n}=60$ ) was remarkably lower than control $(0.55+0.15, \mathrm{n}=60)$, with a $\mathrm{P}<0.01$ (Student's $t$ test). The mean value of skin carotenoids of thalassemia patients was around one half with respect to healthy control (Figure 2). Relationships between the SCS of patients and serum both ferritin, as a marker of iron burden, and ALT and AST, as an index of hepatic damage,

Table 1. Hematological and instrumental data of $\beta$-thalassemia patients.

Pre-transfusion hemoglobin (g/dL) 9.7+1.1

Ferritin $(\mathrm{ng} / \mathrm{mL}) \quad 1439 \pm 961$

AST (U/L) $\quad 35.27 \pm 20.74$

ALT (U/L) $\quad 40.55 \pm 30.25$

Heart T2* (ms) 35.58 $\quad 310.49$

Hepatic T2* (ms) $\quad 4.87 \pm 4.68$

Liver FibroScan ${ }^{\circledR}(\mathrm{kPa})$

$7.55 \pm 5.02$

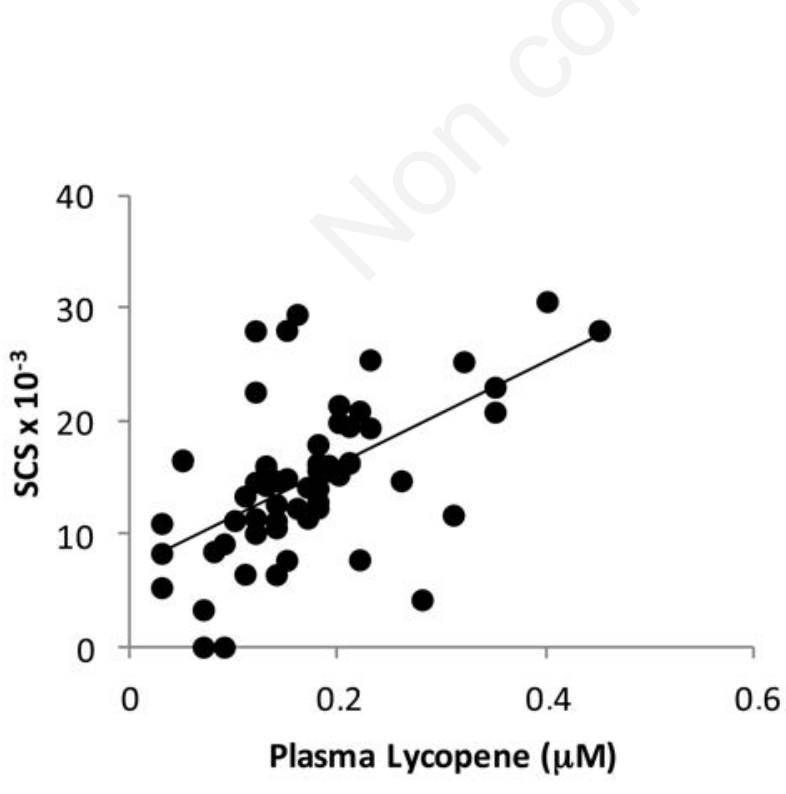

Figure 1. Correlation between plasma lycopene level and skin carotenoid score (SCS) in $\beta$-thalassemia patients. The SCS values and plasma lycopene are the mean of two measurements during the year of the study $(n=57 ; r=0.591, P<0.001)$.

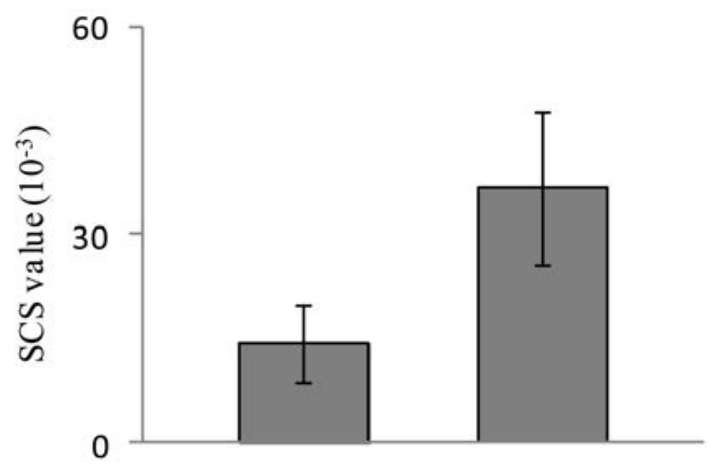

Figure 2. Skin carotenoid score (SCS) in $\beta$-thalassemia patients (left bar) as compared with healthy subjects (control, right bar). Values are the mean \pm SD of two measurements performed on 57 subjects during the year of the study. With respect to the control, values were significant with $\mathrm{P}<0.001$ (Student's t-test). 
were researched. The Figure 3 shows that the SCS had a net inverse correlation with all these parameters. SCS values were correlated with magnetic resonance $\mathrm{T} 2 *$ measurements of both cardiac and hepatic iron (Figure 4). Whereas a positive correlation was found with the cardiac T2* measurements (Figure 4A), no correlation was evident between the level of skin carotenoids and the content of iron in the liver
(Figure 4B). Nevertheless the SCS values showed an inverse correlation with FibroScan ${ }^{\circledR}$ $\mathrm{kPa}$ values from FibroScan ${ }^{\circledR}$ measurements, indicating an inverse relationship with liver stiffness (Figure 5). The SCS values did not show any correlation with the patient age (not shown). As expected, no correlation was found between serum ferritin and $\mathrm{T} 2 *$ measurements of cardiac iron.

\section{Discussion}

Body's low molecular weight antioxidants include a number of vitamins, carotenoids among them. ${ }^{23}$ Because of their physico-chemical characteristics the latter can accumulate in cell membranes. In skin in particular they function as antioxidants and optical absorbers
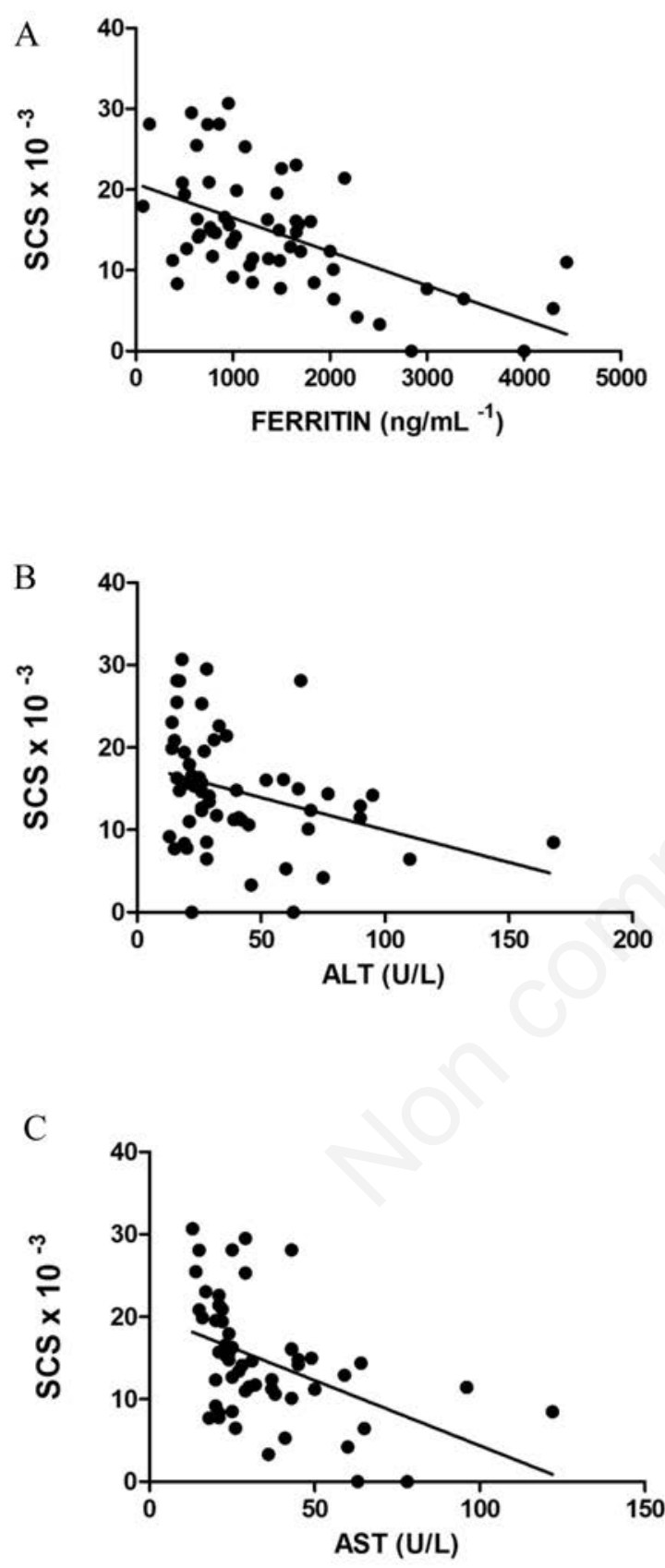

Figure 3. Correlation between skin carotenoid score (SCS) and plasma ferritin (A), alanine (ALT) (B) and aspartate transaminase (AST) (C) in $\beta$-thalassemia patients. Each value is the mean of two measurements during the year of the study. $n=57$; A) $r=-0.569, \quad P<0.0001 ; B) \quad r=-0.292, P=0.027 ; C) \quad r=-0.434$, $\mathbf{P}=\mathbf{0 . 0 0 0 7}$.
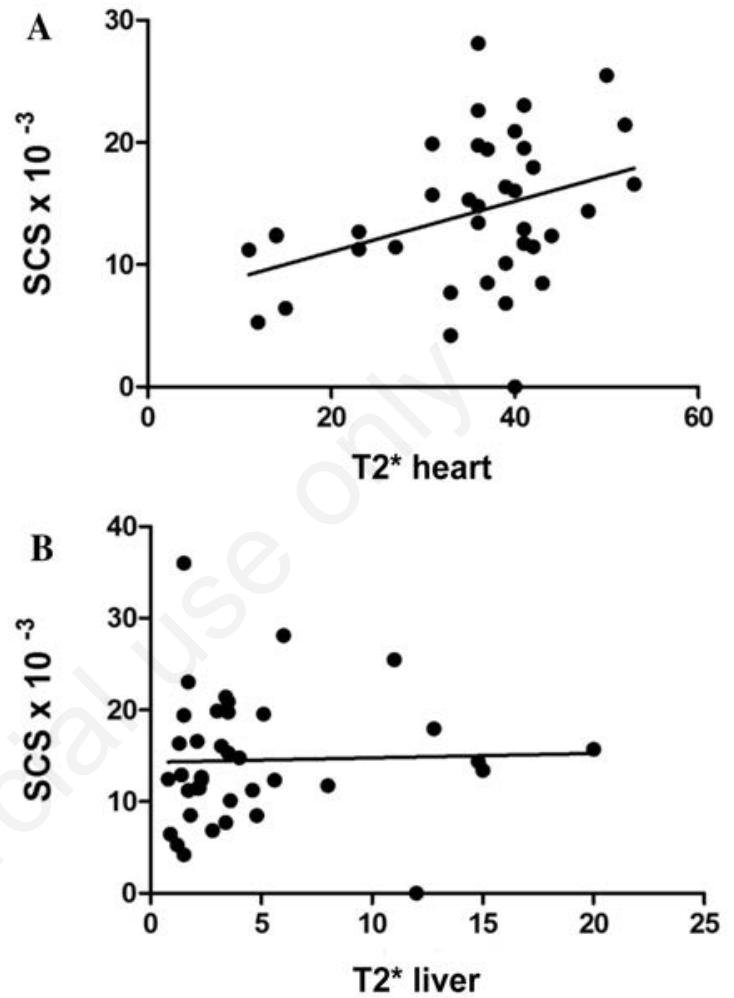

Figure 4. Correlation between skin carotenoid score (SCS) and magnetic resonance (MR) T2* values of the myocardium (A) or liver (B) in $\beta$-thalassemia patients. SCS values are the mean of two measurements during the year of the study. Liver and hearth T2* MR measurements were carried out within the same time interval. $\mathrm{n}=38$; A) $\mathrm{r}=0.364, \mathrm{P}=\mathbf{0 . 0 0 2 4}$; B) $\mathrm{r}=0.176, \mathrm{P}=\mathbf{0 . 2 8 7}$.

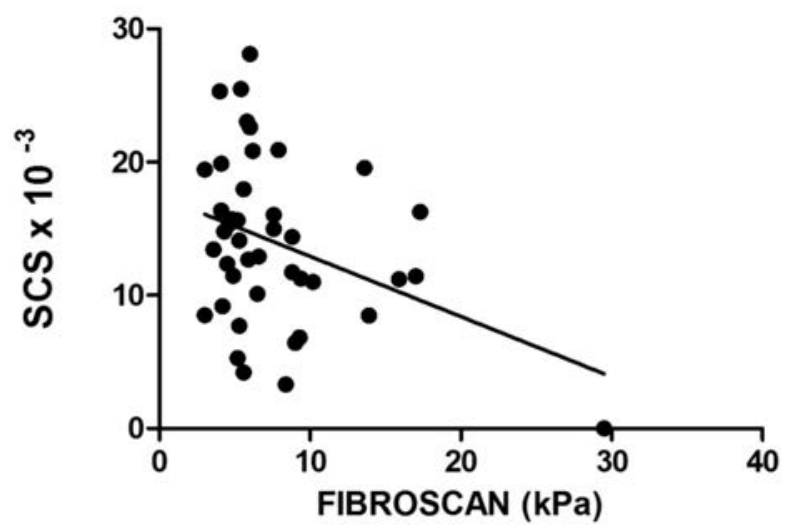

Figure 5. Correlation between skin carotenoid score (SCS) and liver stiffness in $\beta$-thalassemia patients. Liver stiffness was measured by transient elastography (FibroScan $\left.{ }^{\circledR}\right)$. SCS values are the mean of two measurements during the year of the study. FibroScan ${ }^{\circledR}$ examination was carried out within the same time interval $(n=42 ; r=-0.3599, P=0.0192)$. 
of phototoxic light. ${ }^{24-27}$ A novel non-invasive RRS has recently been developed to measure the level of skin carotenoids. ${ }^{7-10}$ This has been validated as a biomarker of the carotenoid status and a reliable probe of the redox balance of healthy humans, ${ }^{9}$ and applied in dietetic studies to monitor body's antioxidant status to eventually suggest dietary interventions..$^{28}$ In this work for the first time the level of dermal carotenoids, measured by Raman spectroscopy and expressed as SCS, has been assessed in patients with a transfusion-dependent thalassemia, and the use of this methodologic approach has been even in this pathology characterized by a remarkable individual oxidative stress. ${ }^{1-3}$

When the SCS of thalassemia patients were correlated with hematologic parameters such as ferritin, ALT and AST, a clear inverse correlation was evident, indicating that the level of skin carotenoids can reliably be representative of the altered both iron status and hepatic pattern characterizing the pathology. As a further indication of clinical opportunity of the SCS in thalassemia, the level of skin carotenoids appeared also inversely associated with the patients' liver fibrosis, as revealed by elastography.

In spite of improvements in the patient care, cardiac iron burden and related morbidity and mortality are still a dreadful problem in transfusion-dependent thalassemia, being a timely detection of the cardiac iron overload and chelation the only prevention and therapy to revert or retard the progression of cardiomyopathy. Serum ferritin has been commonly used for years to evaluate iron overload, however it does not reflect the total storage pool and the level are affected by a variety of conditions. The MR T2* multislice measurements, ${ }^{6}$ is now considered the most accurate non-invasive method of detecting cardiac iron levels and monitoring changes during chelation therapy. ${ }^{29,30}$ In this work the noninvasive measurement of skin carotenoids by RRS has been matched with the cardiac $\mathrm{T} 2 *$ measurements in transfusion-dependent thalassemia patients, and a significant inverse correlation between the SCS and cardiac iron has been found. Then, whereas the relationship between serum ferritin level and heart $\mathrm{T} 2 *$ has been shown to be either non-significant or very weak both in the present and other studies, ${ }^{6,31,32}$ skin carotenoids have appeared to reflect the cardiac iron status of patients. Hopefully measurements on a much larger number of patients will confirm this finding.

When the level of antioxidant carotenoids in skin is considered as the expression of the global redox balance,${ }^{20}$ our measurements confirm the remarkable oxidative stress of transfusion-dependent thalassemia patients, showing that they had a mean loss of more than $50 \%$ of the antioxidant defense. Thalassemia oxidative stress has appeared positively correlated with serum ferritin and liver damage. ${ }^{1}$ Present findings show that SCS measurements may provide an evidence of the association between oxidative stress and heart iron.

Apart from cardiomyocytes, in this study we did not find any correlation between the SCS values and liver MR T2* measurements, showing that the liver iron status cannot be predicted by the patients' level of antioxidants. In other terms the body's oxidative stress in these patients reflects the free iron-related hepatic damage, but does not liver iron accumulation.

Due to the highly adverse effects of accumulated iron, monitoring thalassemia patients at risk is essential to timely provide suitable therapy adjustments and guide chelation choices. The novel, simple, noninvasive Raman spectroscopy to monitor dermal carotenoids with high compliance of the patients may be a useful tool for the management of thalassemia patients. The important relation between skin carotenoids and cardiac $\mathrm{T} 2 *$ measurements suggests the SCS value as an interesting parameter to provide information on oxidative stress and heart iron.

\section{References}

1. Livrea MA, Tesoriere L, Pintaudi AM, et al. Oxidative stress and antioxidant status in $\beta$-thalassemia major : iron overload and depletion of lipid-soluble antioxidants. Blood 1996;88:3608-14.

2. Fibach E, Rachmilewitz E. The role of oxidative stress in hemolytic anemia. Curr Mol Med 2008;8:609-19.

3. Waseem F, Khemomal KA, Sajid R. Antioxidant status in $\beta$ thalassemia major: a single-center study. Indian $\mathrm{J}$ Pathol Microbiol 2011;54:761-3.

4. Doroshow JH, Locker GY, Myers CE. Enzymatic defenses of the mouse heart against reactive oxygen metabolites. Alterations produced by doxorubicin. $\mathrm{J}$ Clin Invest 1980;65:128-35.

5. Ferro E, Visalli G, Civa R, et al. Oxidative damage and genotoxicity biomarkers in transfused and untransfused thalassemic subjects. Free Radic Biol Med 2012;53: 1829-37.

6. Anderson LJ, Holden S, Davis B, et al. Cardiovascular T2-star (T2*) magnetic resonance for the early diagnosis of myocardial iron overload. Eur Heart J 2001;22:2171-9.

7. Hatat TR, Scholz TA, Ermakov IV, et al. Non-invasive Raman spectroscopic detection of carotenoids in human skin. J Invest Dermatol 2000;115:441-8.

8. Ermakov IV, Ermakova MR, Gellermann W,
Lademann J. Noninvasive selective detection of lycopene and $\beta$-carotene in human skin using Raman spectroscopy. J Biomed Opt 2004;9:332-8.

9. Ermakov IV, Gellermann W. Validation model for Raman based skin carotenoid detection. Arch Biochem Biophys 2010; 504:40-9.

10. Mayne ST, Cartmel B, Scarmo S, et al. Resonance Raman spectroscopy evaluation of skin carotenoids as a biomarker of carotenoid status for human studies. Arch Biochem Biophys 2013;539:163-70.

11. Brauchle E, Schenke-Layland K. Raman spectroscopy in biomedicine- non-invasive in vitro analysis of cells and extracellular matrix components in tissues. Biotechnol J 2013;8:288-97.

12. Freed S, Hochanadel CJ. Absorption spectrum of $\beta$ carotene in liquid solution at the temperature of liquid nitrogen. Science 1949;110:298.

13. Bernstein PS, Yoshida MD, Katz NB, et al. Raman detection of macular carotenoid pigments in intact human retina. Invest Ophtalmol Vis Sci 1998;39:2003-11.

14. Ermakov IV, McClane RW, Gellermann W, Bernstein PS. Resonant Raman detection of macular pigment levels in the living human retina. Opt Lett 2001;26:202-4.

15. Sharifzadeh M, Zhao DY, Bernstein PS, Gellermann W. Resonance Raman imaging of macular pigment distributions in the human retina. J 0pt Soc Am A Opt Image Sci Vis 2008;25:947-57.

16. Ermakov IV, Ermakova MR, McClane RW, Gellermann W. Resonance Raman detection of carotenoid antioxidants in living human tissues. Opt Lett 2001;26:1179-81.

17. Ermakov IV, Sharifzadeh M, Ermakova M, Gellermann W. Resonance Raman detection of carotenoid antioxidants in living human tissue. J Biomed Opt 2005;10: 064028.

18. Ermakov IV, Sharfzadeh M, Bernstein PS, Gellermann W. Application of resonance Raman spectroscopy to the detection of carotenoids in vivo. In: Landrum JT, ed. Carotenoids - physical, chemical and biological functions and properties. Atlanta: CRC Press; 2009. pp 87-109.

19. Peng YM, Peng YS, Lin Y, et al. Concentrations and plasma-tissue-diet relationships of carotenoids, retinoids , and tocopherols in humans. Nutr Cancer 1995;23:233-46.

20. Meinke MC, Darvin ME, Vollert H, Lademann J. Bioavailability of natural carotenoids in human skin compared to blood. Eur J Pharm Biopharm 2010;76:269-74.

21. Scarmo S, Cartmel B, Lin $\mathrm{H}$, et al. Significant correlations of dermal total carotenoids and dermal lycopene with their respective plasma levels in healthy 
adults. Arch Biochem Biophys 2010;504: 34-9.

22. Kraft NE. Carotenoid reversed-phase high performance liquid chromatography methods: reference compendium. In: Packer $\mathrm{L}$, ed. Methods in enzimology, vol. 213. San Diego, CA: Academic Press; 1992. pp 185205.

23. Stocker R, Frei B. Endogenous antioxidant defences in human blood plasma. In: Sies $\mathrm{H}$, ed. Oxidative stress: oxidants and antioxidants, vol. 213. New York, NY: Academic Press; 1991.

24. Bohm F, Tinkler JH, Truscott TG. Carotenoids protect against cell membrane damage by nitrogen dioxide radical. Clin. Cancer Res 1995;1:98-9.

25. Foote CS, Denny RW. Chemistry of singlet oxygen. VII. Quenching by $\beta$-carotene. J
Am Chem Soc 1968;90:6233-5.

26. Farmillo A, Wilkinson F. On the mechanism of quenching of singlet oxygen in solution. Photochem Photobiol 1973;18: 447-50.

27. Conn PF, Schalch W, Truscott TG. The singlet oxygen and carotenoid interaction. J Photochem Photobiol B 1991;11:41-7.

28. Scarmo S, Henebery K, Peracchio H, et al. Skin carotenoid status measured by resonance Raman spectroscopy as a biomarker of fruit and vegetable intake in preschool children. Eur J Clin Nutr 2012;66:555-60.

29. Kolnagou A, Economides C, Eracleous E, Kontoghiorghes GJ. Low ferritin levels are misleading for detecting cardiac iron overload and increase the risk of cardiomyopathy in thalassemia patients. The importance of cardiac iron overload monitoring using magnetic resonance imaging T2 and T2*. Hemoglobin 2006;30:219-7.

30. Kondur AK, Li T, Vaitkevicius P, Afonso L. Quantification of myocardial iron overload by cardiovascular magnetic resonance imaging $\mathrm{T}^{*}$ and review of the literature. Clin Cardiol 2009;32:55-9.

31. Wood JC, Tyszka TM, Carson S, et al. Myocardial iron loading in transfusiondependent thalassemia and sickle cell disease. Blood 2004;103:1934-6.

32. Tanner MA, Westwood R, Galanello R, Pennell DJ. Baseline findings of a CMR driven randomized controlled trial of iron chelation therapy in thalassemia major. $\mathrm{J}$ Cardiovasc Magn Reson Imaging 2005;7: 31-2. 